\title{
$B$-meson light-cone distribution amplitudes and radiative leptonic decay
}

\author{
Yao Ji*i \\ Institut für Theoretische Physik, Universität Regensburg, D-93040 Regensburg, Germany \\ E-mail: yao.jicur.de
}

We present the recent developments of $B$-meson light-cone distribution amplitudes (LCDAs) including their definition, classification, evolution, equations of monition (EOMs), and modeling. By making use of these recent developments, one particular decay channel $B \rightarrow \gamma \ell v_{\ell}$ is discussed in detail with the most accurate theory predication to date for its branching ratio which has been used to extract the value of $\lambda_{B}-$ the most important $B$-meson parameter in exclusive decays.

XXVII International Workshop on Deep-Inelastic Scattering and Related Subjects - DIS2019 8-12 April, 2019

Torino, Italy

\footnotetext{
*Speaker.

${ }^{\dagger}$ In collaboration with M. Beneke, V. Braun, A. Manashov and Yan-Bing Wei
} 


\section{Introduction}

The $B$-meson light-cone distribution amplitude (LCDA) [1] is the key ingredient in the study of charmless hadronic $B$-decays under the framework of factorization as it provides crucial nonperturbative information on the hadronic state. Although the LCDAs are inaccessible from first principles at present, we can still study their properties such as asymptotic behaviors, evolution, equations of motion and etc.

In this work, we give a brief summary on the recent developments of $B$-meson LCDAs and their applications to the $B$-meson radiative leptonic decay $B \rightarrow \gamma \ell v_{\ell}$. This decay channel is generally viewed as the theoretically cleanest process for extracting the information on the $B$-meson LCDAs with the upcoming Belle-II data.

\section{2. $B$-meson light-cone distribution amplitudes}

The $B$-meson DAs are defined as the matrix elements of the renormalized nonlocal operators composed of an effective heavy quark field $h_{v}(0)$ and light fields separated by light-like distances. The two-particle LCDAs arise from the light cone expansion of the nonlocal operator with $\left|x^{2}\right| \ll$ $1 / \Lambda_{\mathrm{QCD}}^{2}[1,2]:$

$$
\begin{gathered}
\left\langle 0\left|\bar{q}(x) \Gamma[x, 0] h_{v}(0)\right| \bar{B}(v)\right\rangle=-\frac{i}{2} F_{B}(\mu) \operatorname{Tr}\left[\gamma_{5} \Gamma P_{+}\right] \int_{0}^{\infty} d \omega e^{-i \omega(v \cdot x)}\left\{\phi_{+}(\omega, \mu)+x^{2} g_{+}(\omega, \mu)\right\} \\
+\frac{i}{4} F_{B}(\mu) \operatorname{Tr}\left[\gamma_{5} \Gamma P_{+} \not x\right] \frac{1}{v \cdot x} \int_{0}^{\infty} d \omega e^{-i \omega(v \cdot x)}\left\{\left[\phi_{+}-\phi_{-}\right](\omega, \mu)+x^{2}\left[g_{+}-g_{-}\right](\omega, \mu)\right\},
\end{gathered}
$$

where $[x, 0]$ is the Wilson line connecting the light and heavy quark field that ensures gauge invariance. Such terms are always present but will often be omitted for brevity.

Here and below

$$
n_{\mu}=(1,0,0,1), \quad \bar{n}_{\mu}=(1,0,0-1), \quad v_{\mu}=\frac{1}{2}\left(n_{\mu}+\bar{n}_{\mu}\right), \quad(n \cdot \bar{n})=2
$$

where $v_{\mu}$ is the heavy quark velocity, $P_{+}=\frac{1}{2}(1+\gamma)$ projects out the upper components in the heavy quark spinor, $\Gamma$ is an arbitrary Dirac structure containing a $\gamma^{5}$ matrix. $|\bar{B}(v)\rangle$ is the $\bar{B}$-meson state in the heavy quark effective theory (HQET) and $F_{B}(\mu)$ is the decay constant in HQET which is related to the physical $B$-meson decay constant $f_{B}$. The function $\phi_{ \pm}(\omega, \mu)$ is the two-particle leading- and subleading-twist $B$-meson LCDA, respectively [3]. $g_{ \pm}(\omega, \mu)$ is the two-particle twist-4 and twist-5 LCDA, respectively, generated by the nonvanishing transverse momenta of the light quark.

The LCDAs given in Eq. (2.1) are in the momentum space and are related to the the coordinate space DAs by Fourier transform

$$
\phi_{ \pm}(\omega, \mu)=\frac{1}{2 \pi} \int_{-\infty}^{\infty} d z e^{i \omega z} \Phi_{ \pm}(z-i \varepsilon, \mu), \quad \Phi_{ \pm}(z, \mu)=\int_{0}^{\infty} d \omega e^{-i \omega z} \phi_{ \pm}(\omega, \mu),
$$

with the generalization to DAs of higher Fock states evident. The coordinate space LCDAs are analytic functions of $z$ in the lower half-plane, $\operatorname{Im}(z)<0$. Note that the upper and lower case letters are reserved for the coordinate-space and momentum-space distributions, respectively. 
The three-particle $B$-meson LCDAs are defined similarly by Lorentz decomposition of the three-particle matrix element $\left\langle 0\left|\bar{q}\left(n z_{1}\right) g G_{\mu v}\left(n z_{2}\right) \Gamma h_{v}(0)\right| \bar{B}(v)\right\rangle$. This yields eight independent structures from which we obtain eight LCDAs [4]. For practical purposes, the gluon field strength tensor $G_{\mu \nu}$ is often contracted with a light-like vector reducing the total number of three-particle LCDAs to six [5]. The LCDAs defined in this manner are simple w.r.t the Lorentz structures. They are not, however, convenient for QCD factorization studies as the DAs in this basis have no definite collinear twist. It is therefore instructive to rewrite the three-particle LCDAs in terms of the collinear twist $t=d-s$ and conformal spin $j=\frac{1}{2}(d+s)$ with $d$ being the canonical dimension of the field and $s$ the light-cone spin projection [2] . Physically, the twist $t$ determines the power counting in high-energy processes while the conformal spin $j$ dictates the evolution of the LCDA as well as its asymptotic behavior at small momenta.

The LCDAs are scale-dependent objects which satisfy the renormalization group equation (RGE). The one-loop evolution kernel for the twist-2 DA $\phi_{+}$can be found in Ref. [6] and the evolution kernels for the three-particle LCDAs in coordinate (momentum) space can be obtained from Refs. [7, 8] ([9]). Recently, the two-loop evolution kernel for $\phi_{+}$has become available [10].

It was discovered that the evolution equation for $\phi_{+}$can be solved analytically $[11,12]$. Later, it was demonstrated that the twist- 3 and twist 4 LCDAs are also solvable at the large $N_{c}$ limit $[2,13]$ by making use of a "hidden" symmetry of the RGE called complete integrability [14].

The higher twist LCDAs are not completely independent as they are related to one another by the following equations of motion (EOMs) at tree-level [2, 5],

$$
\begin{aligned}
{\left[z \frac{d}{d z}+1\right] \Phi_{-}(z) } & =\Phi_{+}(z)+2 z^{2} \int_{0}^{1} u d u \Phi_{3}(z, u z) \\
2 z^{2} \mathrm{G}_{ \pm}(z) & =-\left[z \frac{d}{d z}-\frac{1}{2}+i z \bar{\Lambda}\right] \Phi_{ \pm}(z)-\frac{1}{2} \Phi_{\mp}(z)-z^{2} \int_{0}^{1} \bar{u} d u \Psi_{4 / 5}(z, u z) \\
\Phi_{-}(z) & =\left(z \frac{d}{d z}+1+2 i z \bar{\Lambda}\right) \Phi_{+}(z)+2 z^{2} \int_{0}^{1} d u\left[u \Phi_{4}(z, u z)+\Psi_{4}(z, u z)\right]
\end{aligned}
$$

where $\bar{\Lambda}=m_{B}-m_{b}$. The subscript of each three-particle DA specifies its twist.

It is clear from Eqs. (2.4) that the higher-twist two-particle DAs are completely fixed by the three-particle DAs and the leading twist DA, and the combination of Eq. (2.4a) and (2.4b) provides a nontrivial constraint on the three-particle DA.

Neglecting the four-particle DAs systematically due to their high Fock state nature and autonomous evolution at one-loop order, the Lorentz symmetry dictates another relation on twist-4 three-particle DAs [2],

$$
\left[\psi_{4}+\widetilde{\psi}_{4}\right]\left(\omega_{1}, \omega_{2}\right)-\omega_{2} \frac{\partial}{\partial \omega_{2}}\left[\psi_{4}+\widetilde{\psi}_{4}\right]\left(\omega_{1}, \omega_{2}\right)=-2 \omega_{1} \frac{\partial}{\partial \omega_{1}} \phi_{4}\left(\omega_{1}, \omega_{2}\right) .
$$

\section{3. $B$-meson radiative leptonic decay}

The $B$-meson radiative leptonic decay $B \rightarrow \gamma \ell v_{\ell}$ is the cleanest probe for accessing the lightcone structure of the charged $B$-meson if the outgoing photon is highly energetic $E_{\gamma} \gg \Lambda_{\mathrm{QCD}}$. The decay amplitude reads,

$$
\mathscr{A}\left(B^{-} \rightarrow \gamma \ell \bar{v}_{\ell}\right)=\frac{G_{F} V_{u b}}{\sqrt{2}}\left\langle\ell \bar{v}_{l} \gamma\left|\bar{\ell} \gamma^{v}\left(1-\gamma_{5}\right) \nu_{\ell} \bar{u} \gamma_{v}\left(1-\gamma_{5}\right) b\right| B^{-}\right\rangle .
$$


In case of the final state photon is emitted from the $B$-meson constituent, the amplitude can be studied through the following hadronic tensor (correlation function) [15],

$$
\begin{aligned}
T_{\mu v}(p, q) & =-i \int d^{4} x e^{i p x}\left\langle 0\left|T\left\{j_{\mu}^{e m}(x) \bar{u}(0) \gamma_{v}\left(1-\gamma_{5}\right) b(0)\right\}\right| B^{-}(p+q)\right\rangle \\
& =\varepsilon_{\mu \nu \tau \rho} p^{\tau} v^{\rho} F_{V}+i\left[-g_{\mu v}(p v)+v_{\mu} p_{v}\right] F_{A}-i \frac{v_{\mu} v_{v}}{(p v)} f_{B} m_{B}+p_{\mu} \text {-terms. }
\end{aligned}
$$

where the Lorentz decomposition is applied to obtain the second equation from which two form factors $F_{V}$ and $F_{A}$ are defined. $p$ and $q$ are the photon and lepton-pair momenta, respectively so that $(p+q)=m_{B} v$ is the $B$-meson momentum in its rest frame. $j_{\mu}^{e m}$ is the electromagnetic current generated by the quarks. The coefficient of the contact term $v_{\mu} v_{v}$ is fixed by the Ward identity. Terms that are proportional to $p_{\mu}$ do not contribute to the decay amplitude by contracting with the photon polarization vector.

The differential decay width is then given in terms of the two form factors by [15]

$$
\frac{d \Gamma}{d E_{\gamma}}=\frac{\alpha_{\mathrm{em}} G_{F}^{2}\left|V_{u b}\right|^{2}}{6 \pi^{2}} m_{B} E_{\gamma}^{3}\left(1-\frac{2 E_{\gamma}}{m_{B}}\right)\left(\left|F_{V}\right|^{2}+\left|F_{A}+\frac{e_{\ell} f_{B}}{E_{\gamma}}\right|^{2}\right)
$$

where the leptonic photon emission has been included.

For large photon energies, the form factors can be written as [15]

$$
F_{V / A}\left(E_{\gamma}\right)=\frac{e_{u} f_{B} m_{B}}{2 E_{\gamma} \lambda_{B}(\mu)} R\left(E_{\gamma}, \mu\right)+\xi\left(E_{\gamma}\right) \pm \Delta \xi\left(E_{\gamma}\right)
$$

The first term is the leading-power contribution generated by $\phi_{+}$with the quark propagator in the hard-collinear region, i.e., $1 / x^{2} \gg \Lambda_{\mathrm{QCD}}^{2}$ in Eq. (3.1). The QCD corrections to the leading power contribution are factorized into $R\left(E_{\gamma}, \mu\right)$ to all orders in $\alpha_{s}[16,17]$ with the $\mathscr{O}\left(\alpha_{s}\right)$ result available in Ref. [15]. $\lambda_{B}(\mu)$, which is defined as

$$
\frac{1}{\lambda_{B}(\mu)}=\int_{0}^{\infty} \frac{d \omega}{\omega} \phi_{+}(\omega, \mu)
$$

is the most important $B$-meson LCDA parameter in exclusive $B$-meson decays. The remaining terms in Eq. (3.4) denote the power-suppressed $1 /\left(2 E_{\gamma}\right)$ and $1 / m_{b}$ corrections with $\xi\left(E_{\gamma}\right)\left(\Delta \xi\left(E_{\gamma}\right)\right)$ being the "symmetry preserving (breaking)" part. To our accuracy, $\xi\left(E_{\gamma}\right)$ and $\Delta \xi\left(E_{\gamma}\right)$ read [18],

$$
\xi=\xi^{\mathrm{ht}}+\xi_{(\mathrm{NLO})}^{\mathrm{soft}}+\xi_{(t w-3,4)}^{\mathrm{soft}}+\xi_{(t w-5,6)}^{\mathrm{soft}}, \quad \Delta \xi=\Delta \xi^{\mathrm{ht}}+\Delta \xi_{(t w-3,4)}^{\mathrm{soft}}+\Delta \xi_{(t w-5,6)}^{\mathrm{soft}} .
$$

Here $\xi^{\text {ht }}$ and $\Delta \xi^{\text {ht }}$ are generated by twist-3 and 4 LCDAs in the hard-collinear region whereas $\xi_{(t w-3,4)}^{\text {soft }}$ and $\Delta \xi_{(t w-3,4)}^{\text {soft }}$ arise when $1 / x^{2} \lesssim \Lambda_{\mathrm{QCD}}^{2}$ in Eq. (3.1). In the latter case, the light-cone factorization breaks down and in principle, LCDAs of all twists can contribute at the same power in the $1 / E_{\gamma}$ expansion. It is, therefore, impossible to include such contributions without additional assumptions. To this end, we adopt the light-cone sum rules (LCSR) [19]. $\xi_{\text {(NLO) }}^{\text {soft denotes the }}$ soft correction to the leading power contribution to $\mathscr{O}\left(\alpha_{s}\right)[20] . \xi_{(t w-5,6)}^{\text {soft }}$ and $\Delta \xi_{(t w-5,6)}^{\text {soft }}$ are generated by twist- 5 and 6 DAs that can be factorized into a product of lower-twist DAs and the quark condensate. The relative size of these contributions to their twist- 3 and 4 counterparts indicates whether the twist expansion in the soft region is converging. 
The explicit expressions for each contribution in Eq. (3.6) are available in Ref. [18] and we will not repeat them here. It is, however, important to note that $\xi^{\text {ht }}$ does not have any end-point divergences due to nontrivial cancellations between different higher-twist contributions and EOMs (2.4). It is certainly interesting to understand this observation in the context of a factorization theorem.

In order to make numerical predictions for the decay width (3.3), we construct a general anstaz for the higher-twist LCDAs which satisfy the constraints in Eqs. (2.4) and (2.5), and the normalization conditions of the LCDAs (see Eq. (A.39) in [18] for details). Previous models for the higher-twist LCDAs $[2,21]$ become special cases of the general anstaz. It is interesting to note that the general ansatz allows us to obtain $\xi^{\text {ht }}$ without knowing the explicit form of the LCDAs [18].

The soft-corrections, however, require explicit expression for $\phi_{+}(\omega, \mu)$. To this end, we have proposed three models [18] motivated by recent pion studies [22, 23, 24, 25]. Note that we always assume the "true" LCDAs are obtained by adding a proper high momentum tail to our models such that $\lambda_{B}$ and other inverse logarithmic moments are independent of the 1st and 2nd moment of $\phi_{+}$[18]. We are then ready to make predictions for the $B \rightarrow \gamma \ell v_{\ell}$ branching ratio with $E_{\gamma} \gg \Lambda_{\mathrm{QCD}}$.
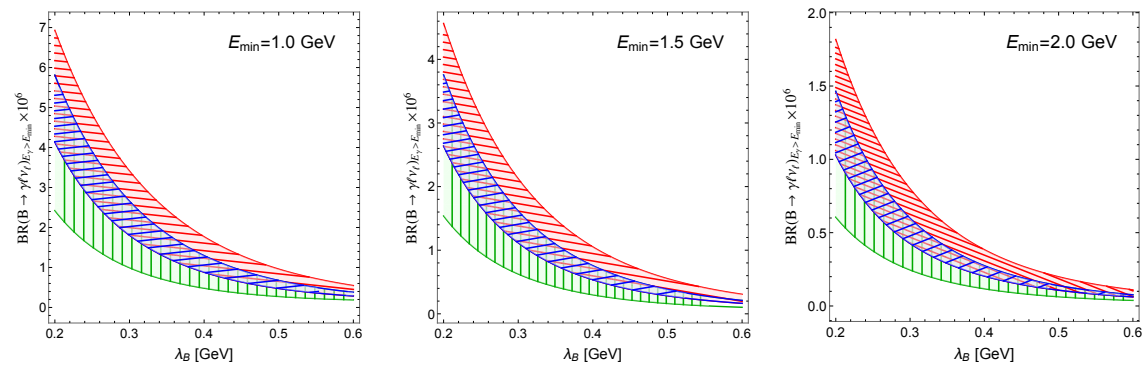

Figure 1: Integrated partial branching fraction $\mathrm{BR}\left(B \rightarrow \gamma \ell v_{\ell}, E_{\gamma}>E_{\min }\right)$ for $E_{\min }=1 \mathrm{GeV}$ (left), $E_{\min }=$ $1.5 \mathrm{GeV}$ (middle) and $E_{\min }=2 \mathrm{GeV}$ (right).

The result is shown in Fig. 1 as a function of $\lambda_{B}$ for three values of the photon low-energy cutoffs with color coding referring to the region covered by different models [18]. The overall envelope of all three bands reflects the total dependence of the branching ratio to the inverselogarithmic moment. The total uncertainty due to input parameters is very minor at $6-9 \%$ level.

Recently, the Belle collaboration applied the prediction in Fig. 1 to determine $\lambda_{B}=0.36_{-0.09}^{+0.25} \mathrm{GeV}$ with $\lambda_{B}>0.24 \mathrm{GeV}$ at $90 \% \mathrm{CL}$ [26] consistent with theory prediction for $\lambda_{B}$ [27]. It is important to note, however, that this estimate is obtained from extrapolating the low energy data of $E_{\gamma}$ and therefore additional uncertainties may be present.

\section{Summary and outlook}

The $B$-meson LCDAs are the nonperturbative ingredient for exclusive $B$-decays with many interesting properties. The recent developments of higher-twist DAs enable us to study many $B$ decays with higher theory accuracy $([18,28,29,30,31])$. From the theory prediction in Ref. [18], a more precise value of $\lambda_{B}$ has been extracted. We have shown that the higher-twist contribution to the $B \rightarrow \gamma \ell v_{\ell}$ decay is free of end-point divergence which invites further studies in the perspective 
of factorization theorem. We demonstrated that the first inverse logarithmic moment has nonnegligible effect on the $B \rightarrow \gamma \ell v_{\ell}$ branching ratio. The recent result for the two-loop kernel of $\phi_{+}[10]$ calls for studies at the full next-to-next-to-leading-logarithmic accuracy.

\section{References}

[1] A. G. Grozin and M. Neubert, Phys. Rev. D 55 (1997) 272.

[2] V. M. Braun, Y. Ji and A. N. Manashov, JHEP 1705 (2017) 022.

[3] M. Beneke and T. Feldmann, Nucl. Phys. B 592 (2001) 3.

[4] B. Geyer and O. Witzel, Phys. Rev. D 72 (2005) 034023.

[5] H. Kawamura, J. Kodaira, C. F. Qiao and K. Tanaka, Phys. Lett. B 523 (2001) 111 Erratum: [Phys. Lett. B 536 (2002) 344].

[6] B. O. Lange and M. Neubert, Phys. Rev. Lett. 91 (2003) 102001.

[7] V. M. Braun, A. N. Manashov and J. Rohrwild, Nucl. Phys. B 826 (2010) 235.

[8] M. Knodlseder and N. Offen, JHEP 1110 (2011) 069.

[9] Y. Ji and A. V. Belitsky, Nucl. Phys. B 894 (2015) 161.

[10] V. M. Braun, Y. Ji and A. N. Manashov, arXiv:1905.04498 [hep-ph].

[11] G. Bell, T. Feldmann, Y. M. Wang and M. W. Y. Yip, JHEP 1311 (2013) 191.

[12] V. M. Braun and A. N. Manashov, Phys. Lett. B 731 (2014) 316.

[13] V. M. Braun, A. N. Manashov and N. Offen, Phys. Rev. D 92 (2015) no.7, 074044.

[14] V. M. Braun, Y. Ji and A. N. Manashov, JHEP 1806 (2018) 017.

[15] M. Beneke and J. Rohrwild, Eur. Phys. J. C 71 (2011) 1818.

[16] E. Lunghi, D. Pirjol and D. Wyler, Nucl. Phys. B 649 (2003) 349.

[17] S. W. Bosch, R. J. Hill, B. O. Lange and M. Neubert, Phys. Rev. D 67 (2003) 094014.

[18] M. Beneke, V. M. Braun, Y. Ji and Y. B. Wei, JHEP 1807 (2018) 154.

[19] V. M. Braun and A. Khodjamirian, Phys. Lett. B 718 (2013) 1014.

[20] Y. M. Wang, JHEP 1609 (2016) 159.

[21] A. Khodjamirian, T. Mannel and N. Offen, Phys. Rev. D 75 (2007) 054013.

[22] S. S. Agaev, V. M. Braun, N. Offen and F. A. Porkert, Phys. Rev. D 83 (2011) 054020.

[23] S. S. Agaev, V. M. Braun, N. Offen and F. A. Porkert, Phys. Rev. D 86 (2012) 077504.

[24] I. C. Cloët, L. Chang, C. D. Roberts, S. M. Schmidt and P. C. Tandy, PRL 111 (2013) 092001.

[25] N. G. Stefanis and A. V. Pimikov, Nucl. Phys. A 945 (2016) 248.

[26] M. Gelb et al. [Belle Collaboration], Phys. Rev. D 98 (2018) no.11, 112016.

[27] V. M. Braun, D. Y. Ivanov and G. P. Korchemsky, Phys. Rev. D 69 (2004) 034014.

[28] C. D. Lü, Y. L. Shen, Y. M. Wang and Y. B. Wei, JHEP 1901 (2019) 024.

[29] N. Gubernari, A. Kokulu and D. van Dyk, JHEP 1901 (2019) 150.

[30] Y. M. Wang and Y. L. Shen, JHEP 1805 (2018) 184.

[31] Y. L. Shen, Z. T. Zou and Y. B. Wei, Phys. Rev. D 99 (2019) no.1, 016004. 